\title{
Genomic analysis of small nucleolar RNAs identifies distinct molecular and prognostic signature in hepatocellular carcinoma
}

\author{
HONG YANG ${ }^{1}$, PENG LIN ${ }^{1}$, HUA-YU WU ${ }^{2}$, HAI-YUAN LI ${ }^{1}$, YUN HE ${ }^{1}$, \\ YI-WU DANG ${ }^{3}$ and GANG CHEN ${ }^{3}$ \\ ${ }^{1}$ Department of Medical Ultrasonics, The First Affiliated Hospital of Guangxi Medical University; \\ ${ }^{2}$ Department of Cell Biology and Genetics, School of Pre-Clinical Medicine, Guangxi Medical University; \\ ${ }^{3}$ Department of Pathology, The First Affiliated Hospital of Guangxi Medical University, \\ Nanning, Guangxi 530021, P.R. China
}

Received May 15, 2018; Accepted September 3, 2018

DOI: $10.3892 /$ or.2018.6715

\begin{abstract}
As one of the most lethal malignancies worldwide, hepatocellular carcinoma (HCC) has a high mortality rate, which is mainly due to the complex and multi-step aberrations in gene expression associated with it. Small nucleolar RNAs (snoRNAs), non-coding RNAs that are 60-300 nucleotides in length, have been proposed to be closely associated with numerous human diseases, including HCC. However, the current knowledge regarding their clinical significance and mechanistic roles in HCC is limited. The present study comprehensively analyzed the snoRNA expression profiles in HCC and identified several ones that were dysregulated. The potential regulatory mechanisms of these snoRNAs were assessed via gene functional enrichment analyses. Univariate and multivariate Cox regression analyses were performed to identify snoRNAs that are independently associated with the risk of mortality. Subsequently, a prognostic index (PI) for survival prediction was established, which may serve as a prognostic biomarker for patients with HCC (hazard ratio, 3.023; 95\% confidence interval: 1.785-5.119; $\mathrm{P}<0.001)$. In addition, a series of bioinformatics analyses were performed to identify potential differences in the perturbation of pathways between high- and low-risk groups. The PI developed in the present study was determined to have a moderate predictive value regarding the clinical outcome for HCC patients.
\end{abstract}

Correspondence to: Professor Gang Chen, Department of Pathology, The First Affiliated Hospital of Guangxi Medical University, 6 Shuangyong Road, Nanning, Guangxi 530021, P.R. China

E-mail: chengang@gxmu.edu.cn

Key words: hepatocellular carcinoma, small nucleolar RNAs, prognostic index, personalized medicine, The Cancer Genome Atlas

\section{Introduction}

Liver cancer ranks as one of the most lethal malignancies worldwide, with 42,220 new cases and 30,200 deaths estimated for the United States for 2018 (1). Hepatocellular carcinoma (HCC) is the predominant histological subtype of liver cancer and places a heavy burden on human health $(2,3)$. HCC is associated with multiple etiological factors, including hepatitis $\mathrm{C}$ and $\mathrm{B}$ virus infection, exposure to toxins and alcohol abuse $(4,5)$. Therapeutic strategies combining surgical resection and molecular targeted treatment have provided encouraging results and improved the outcomes for HCC patients. However, the prognosis for patients with unresectable advanced-stage HCC remains poor $(6,7)$. Sorafenib was the only systemic drug available for treating advanced HCC until the recent approval of regorafenib, another multi-kinase inhibitor $(8,9)$. However, sorafenib and regorafenib have a low durable response rate and their benefit for survival is limited (10). Hence, novel prognostic biomarkers and a deeper understanding of the exact molecular mechanisms in HCC are urgently required to improve its clinical management.

While it was previously assumed that genes encoding non-coding RNAs have no function, accumulating evidence has proved that several non-coding RNAs, including long non-coding RNA (lncRNA) and microRNA (miRNA), have vital regulatory roles in cellular biology and physiological processes. Of note, small nucleolar RNAs (snoRNAs), which are non-coding RNAs that are 60-300 nucleotides in length, were proposed to be closely associated with various human diseases, including cancer $(11,12)$. Several studies have reported that certain snoRNAs act as diagnostic or prognostic biomarkers and as therapeutic targets for $\mathrm{HCC}(13,14)$. Several in vitro and in vivo studies indicated that certain snoRNAs are involved in the regulation of the genesis and biological behavior of HCC, including cell proliferation, migration, apoptosis, cell cycle and metastasis. In spite of this, the function of snoRNAs in HCC remains limited and requires further elucidation. Systematic investigation of the expression profiles and clinical significance of snoRNAs in HCC may 
provide a deeper understanding of their roles in $\mathrm{HCC}$ and contribute to the development of novel therapeutic strategies. Gong et al (15) developed an online database of snoRNAs in cancers (SNORic), which provides expression profiles in $>10,000$ samples of different tumor types using calculations based on The Cancer Genome Atlas (TCGA) database. The database provides expression profiles of snoRNAs for analysis.

The present study comprehensively analyzed differentially expressed snoRNAs in HCC and provided an overview of their clinical significance. Subsequently, several functional enrichment analyses were performed to elucidate the functional roles of key snoRNAs. More importantly, survival-associated snoRNAs were identified to develop a prognostic index (PI), which may be utilized as a risk score model for HCC patients. Via these efforts, the present study aimed to propose a foundation and comprehensive view of snoRNAs in HCC and identify novel biomarkers to effectively predict clinical outcomes.

\section{Materials and methods}

Data preparation and pre-processing. The snoRNA gene expression profiles of 1,524 HCC patients were downloaded from the online database SNORic (http://bioinfo.life.hust.edu. $\mathrm{cn} / \mathrm{SNORic} /$ ) (15). The expression value of snoRNAs was normalized and quantified as reads per kilobase per million mapped reads (RPKM). Only snoRNAs with an average RPKM of $>1$ across all samples were used for further analysis. The corresponding clinical information of the HCC patients was also downloaded from the TCGA database (https://portal.gdc.cancer.gov/).

Screening of differentially expressed snoRNAs. Two different strategies, including analysis with the 'limma' package in $\mathrm{R}$ (http://www.bioconductor.org/packages/release/ bioc/html/limma.html) and an independent-samples t-test via SPSS 24.0 (IBM Corp., Armonk, NY, USA), were used to identify snoRNAs which were significantly differentially expressed between HCC and non-tumor tissues. For the limma test, the threshold for the significantly differentially expressed snoRNAs was considered a Ifold changel $\geq 2$ and a false discovery rate (FDR) of $<0.05$. For the t-test, snoRNAs were considered differentially expressed when $\mathrm{P}<0.05$. Genes that were identified by the two differential analyses simultaneously were defined as differentially expressed snoRNAs.

Functional annotation of snoRNAs. To further explore the potential functional roles of the differentially expressed snoRNAs, the top 10 most significantly differentially expressed snoRNAs were selected and messenger RNAs (mRNAs) with expression levels correlated with these snoRNAs were obtained from SNORic. Next, these mRNAs were subjected to functional enrichment analysis using the ClusterProfiler package (16), in order to identify the enrichment of the snoRNAs in various Gene Ontology (GO) categories and Kyoto Encyclopedia of Genes and Genomes (KEGG) processes. ClusterProfiler also calculated corrected P-values to prevent a high FDR in multiple testing. GO and KEGG terms/pathways with corrected P-values of $<0.05$ were considered to be significantly enriched among the associated genes.
Survival analysis of key driver genes. snoRNAs that were candidate prognostic biomarkers were then selected. To obtain more accurate results/avoid immortal-time bias, patients with $<90$ days of overall survival (OS) were removed prior to survival analysis (17). The patients were followed up for a duration of 91-3,675 days. The association between snoRNA expression and OS was performed by using univariate Cox regression. Candidate prognostic snoRNAs were then subjected to multivariate Cox regression. A survival-predicting algorithm PI, an index calculated for each patient according to their snoRNA expression pattern, was built according to the expression values of each independent snoRNA and weighted by the contribution of each snoRNA to OS (18). The 'survivalROC' package in R (https://CRAN.R-project.org/package=survivalROC) was used to evaluate the performance of the algorithm in predicting the prognosis of the HCC patients. The ability of the models (PI) to predict the outcomes was calculated at 2,000 days, as only few events occurred after this time-point.

Gene set enrichment analysis (GSEA). GSEA was performed to analyze the deregulated pathways between patients with a high and low risk according to the predictive model/PI established (19). First, GSEA generated an ordered list of all genes based on their association with the PI. Subsequently, the pre-defined KEGG pathways were calculated with an enrichment score (ES) and nominal P-value. Finally, each pathway was given a normalized ES (NES) and an FDR calculated for the ES. Pathways with NES $>1$ and FDR $<0.05$ were considered significant. The different risk groups served as phenotype labels.

\section{Results}

Identification of differentially expressed snoRNAs. A total of $372 \mathrm{HCC}$ and 50 non-tumor tissues were included in the present analysis. A total of 453 snoRNAs with an average RPKM of $>1$ were obtained. Of these, 133 differentially expressed snoRNAs were assessed using the limma statistical package, including 119 that were upregulated and 14 that were downregulated (Ifold changel $\geq 2$ and FDR $<0.05$ ). As indicated in the volcano plot, most of these differentially expressed snoRNAs were upregulated (Fig. 1). Furthermore, 71 upregulated and 272 downregulated snoRNAs were identified using the t-test. A total of 68 overlapping snoRNAs (54 upregulated and 14 downregulated snoRNAs) between these methods were identified (Table I). In addition, 65 snoRNAs and 275 snoRNAs were identified by either limma analysis only or the t-test only, respectively. Analysis of the chromosomal distribution of the genes encoding the differentially expressed snoRNAs revealed that the genes encoding these snoRNAs are mostly located on chromosome 1 (Fig. 2).

Functional characteristic of snoRNAs in HCC. Functional enrichment analysis of 1,149 mRNAs associated with differentially expressed snoRNAs was performed using clusterProfiler. Biological processes (BP), cell composition (CC) and molecular function (MF) were the three categories of $\mathrm{GO}$ terms. In the BP category, the three most enriched items were 'ribosomal (r)RNA metabolic process', 'rRNA processing' and 'ribosome biogenesis' (Fig. 3A). In the category $\mathrm{CC}$, the 
A

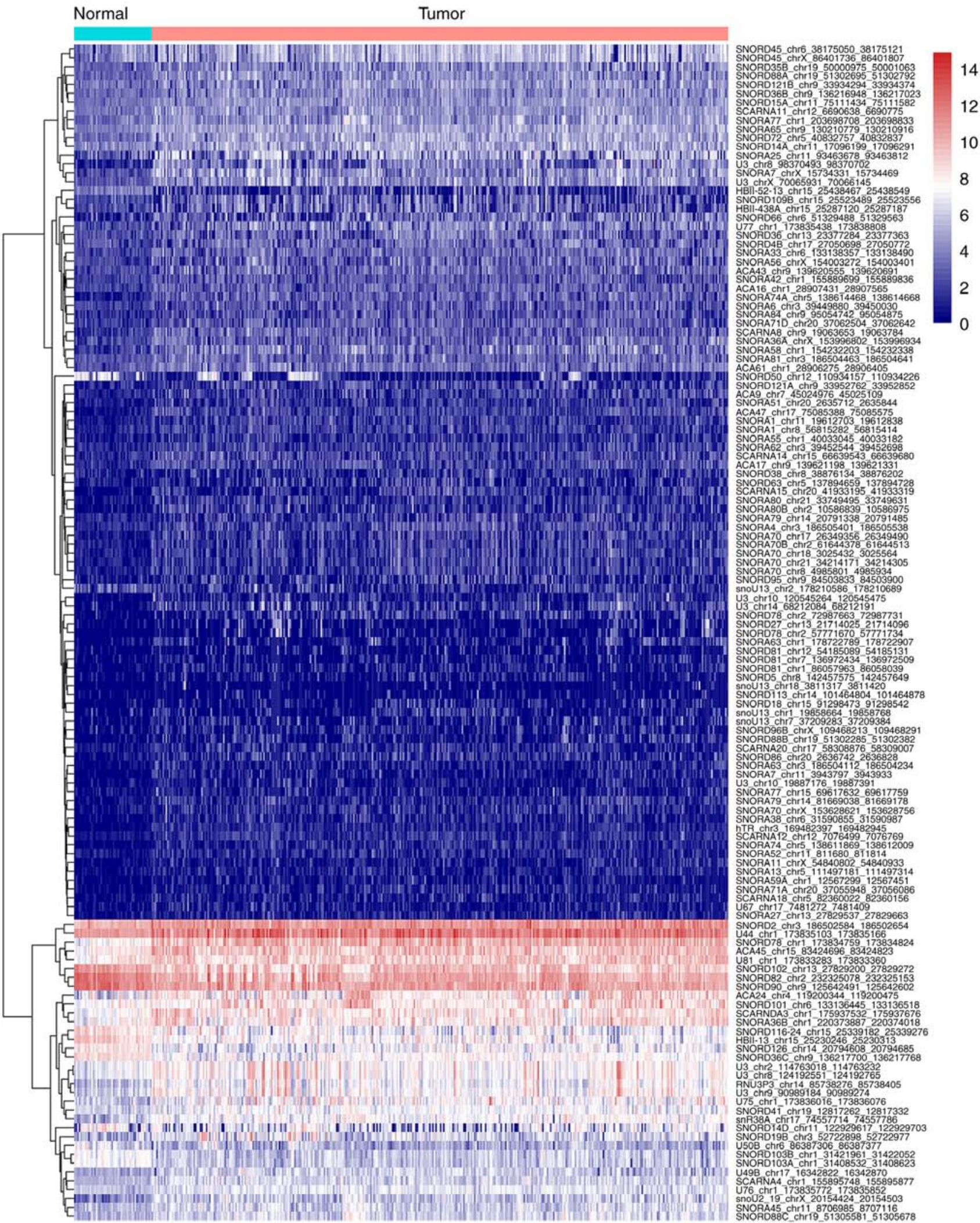

Figure 1. Differentially expressed snoRNAs in hepatocellular carcinoma. (A) Heatmap of the differentially expressed snoRNAs. Blue represents differentially expressed snoRNAs and red represents snoRNAs with no significant difference in expression. snoRNA, small nucleolar RNA; FDR, false discovery rate.

mRNAs were mainly concentrated in the terms 'cytosolic ribosome', 'cytosolic part' and 'ribosomal subunit' (Fig. 3B). 'Structural constituent of ribosome', 'cadherin binding involved in cell-cell adhesion' and 'protein binding involved in cell adhesion' were the more prominent terms enriched by the mRNAs in the MF category (Fig. 3C). More interestingly, KEGG analysis indicated that the mRNAs associated with the HCC-specific snoRNAs were most significantly enriched in the pathways 'Ribosome', 'Cell cycle' and 'DNA replication' (Fig. 4). Among these pathways, 'Ribosome' was 


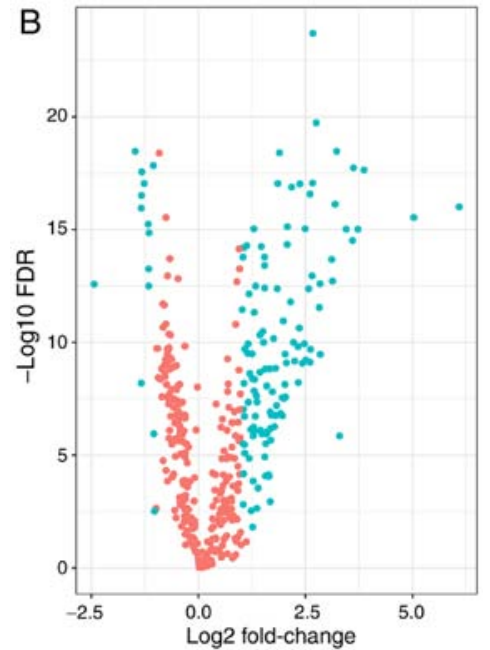

Figure 1. Continued. Differentially expressed snoRNAs in hepatocellular carcinoma. (B) Volcano plot for the differentially expressed snoRNAs. The $\mathrm{x}$-axis indicates the $\log 2$ Ifold changel and the $\mathrm{y}$-axis indicates the $-\log 10$ FDR. Blue represents differentially expressed snoRNAs and red represents snoRNAs with no significant difference in expression. The volcano plot was generated based on the results from the limma package. snoRNA, small nucleolar RNA; FDR, false discovery rate. the most significant pathway and included 42 genes when the background of the functional enrichment analysis was set to 'Homo sapiens' (https://www.kegg.jp/dbget-bin/www_ bget?pathway+hsa03010).

Prognostic predictors for HCC patients. After removing patients with $<90$ days of OS, 330 HCC patients were included in the further analysis. The prognostic value of the differentially expressed snoRNAs was assessed using univariate Cox regression. A total of 22 snoRNAs with $\mathrm{P}<0.05$ were identified, which were therefore able to predict the survival of HCC patients. These snoRNAs were then subjected to multivariate Cox proportional regression analysis, which identified 9 snoRNAs as independent prognostic indicators for HCC. Finally, the PI was calculated based on these 9 snoRNAs as follows: [expression of SNORA (SNOR, H/ACA box)24] x $0.0655+$ (expression of SNORA7) x $0.0991+$ (expression of SNORA63) x $0.1196+$ (expression of U3_chr8-2) x 0.2590 + (expression of U3_chr9) x $0.2464+$ [expression of SNOR, C/D box (SNORD)19B] $\times 0.0613+$ (expression of hTR) $\mathrm{x} 0.1653+($ expression of SNORD36C) $\mathrm{x} 0.0830+($ expression of U44) $x$ 0.0964. The expression of SNORD36C was markedly

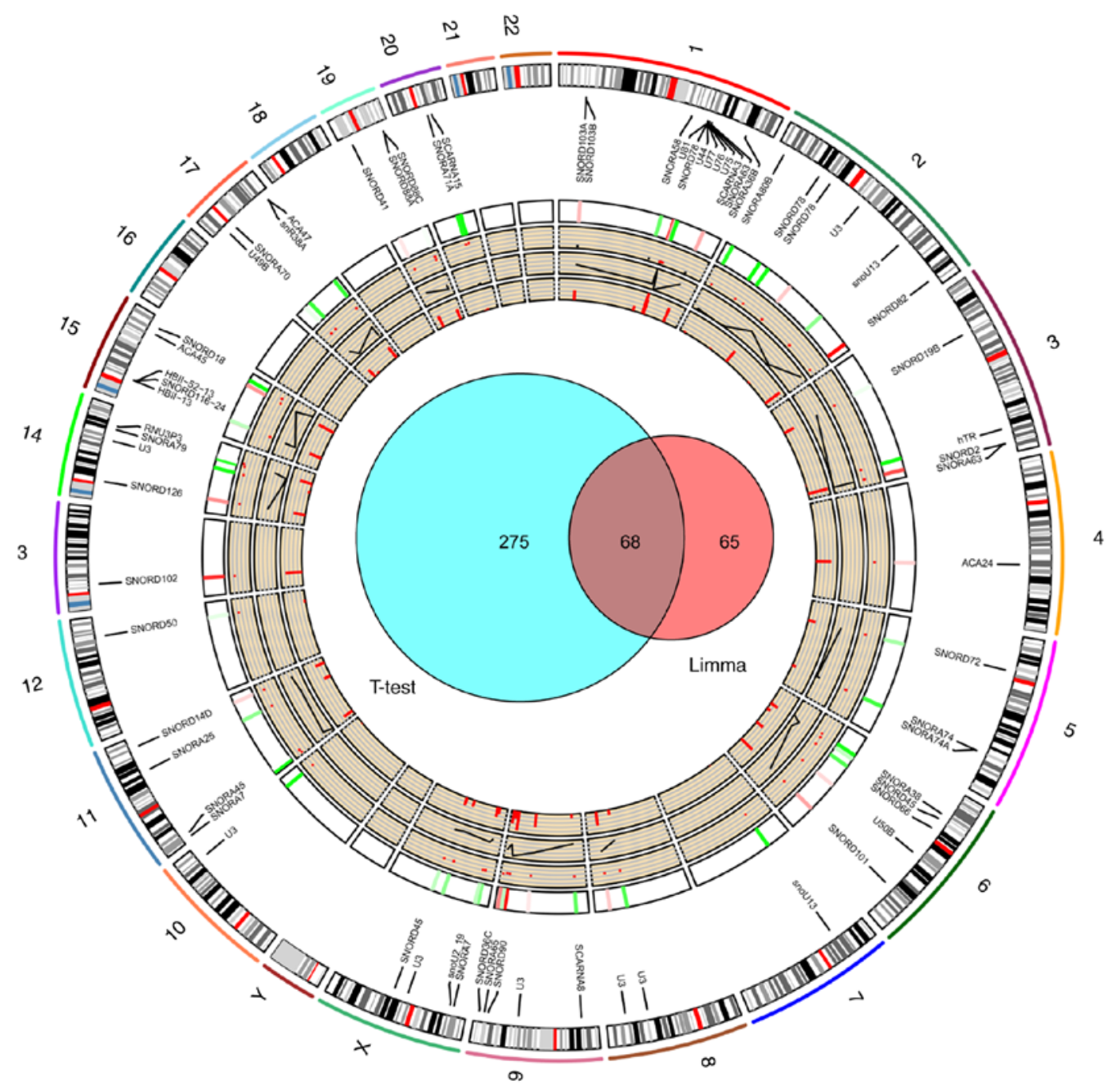

Figure 2. Location of differentially expressed snoRNAs in the genome. The outer ring is the chromosomal ideogram with the chromosome numbers displayed. At the center of the circle, is a Venn diagram is presented. A total of 68 overlapping snoRNAs between limma analysis and the t-test were identified. In addition, 65 snoRNAs and 275 snoRNAs were identified by either limma analysis only or the t-test only, respectively. snoRNA, small nucleolar RNA. 


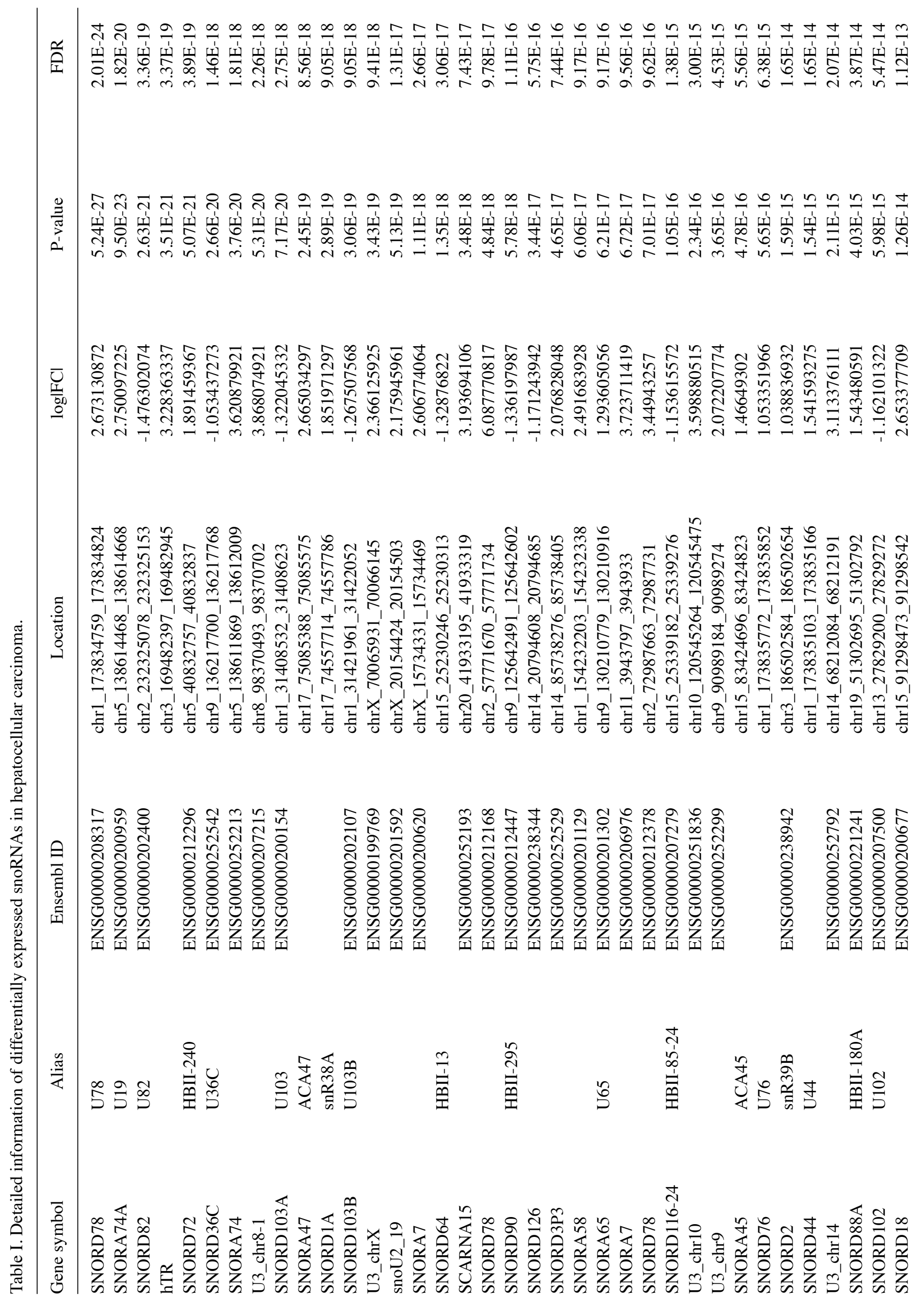




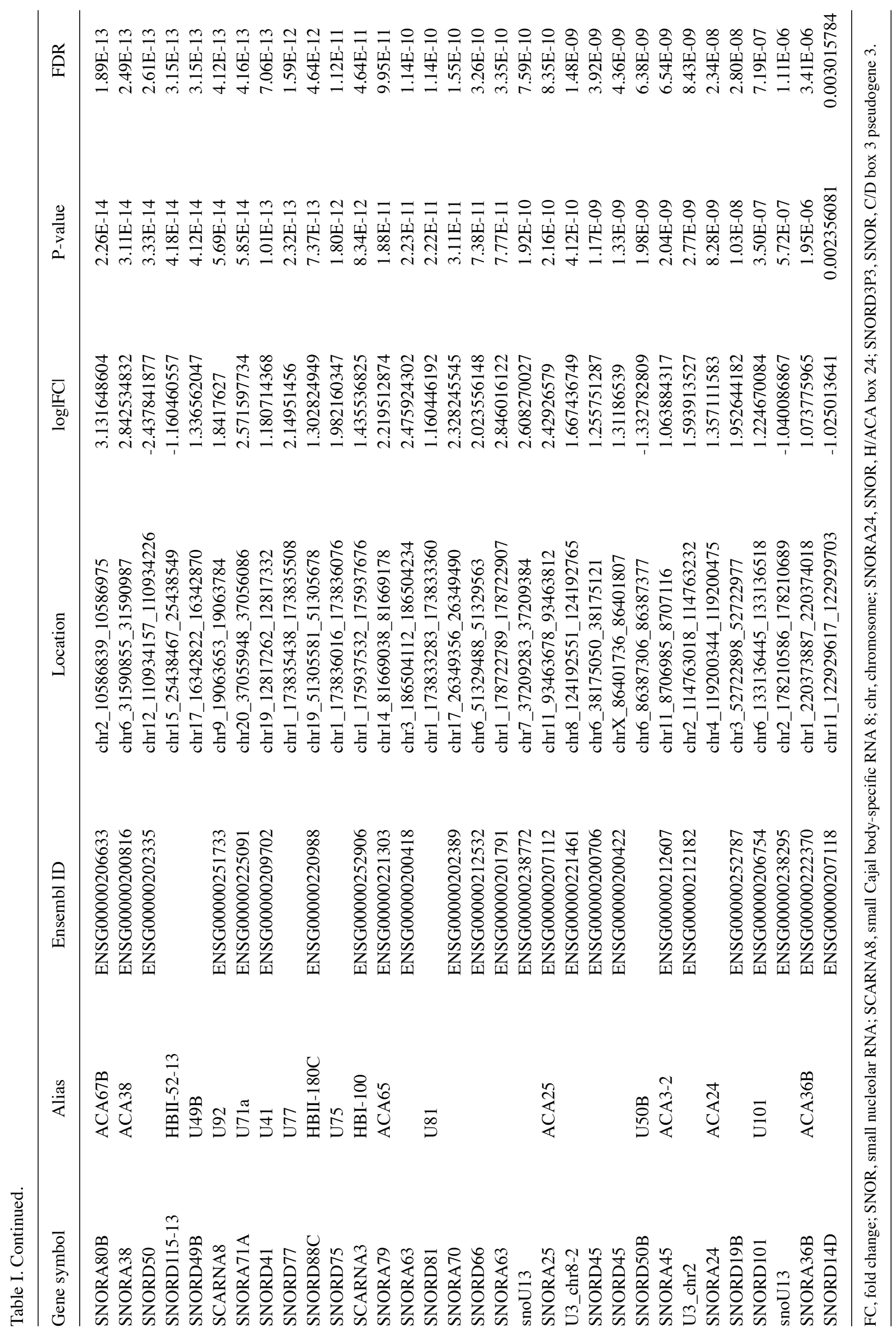


A

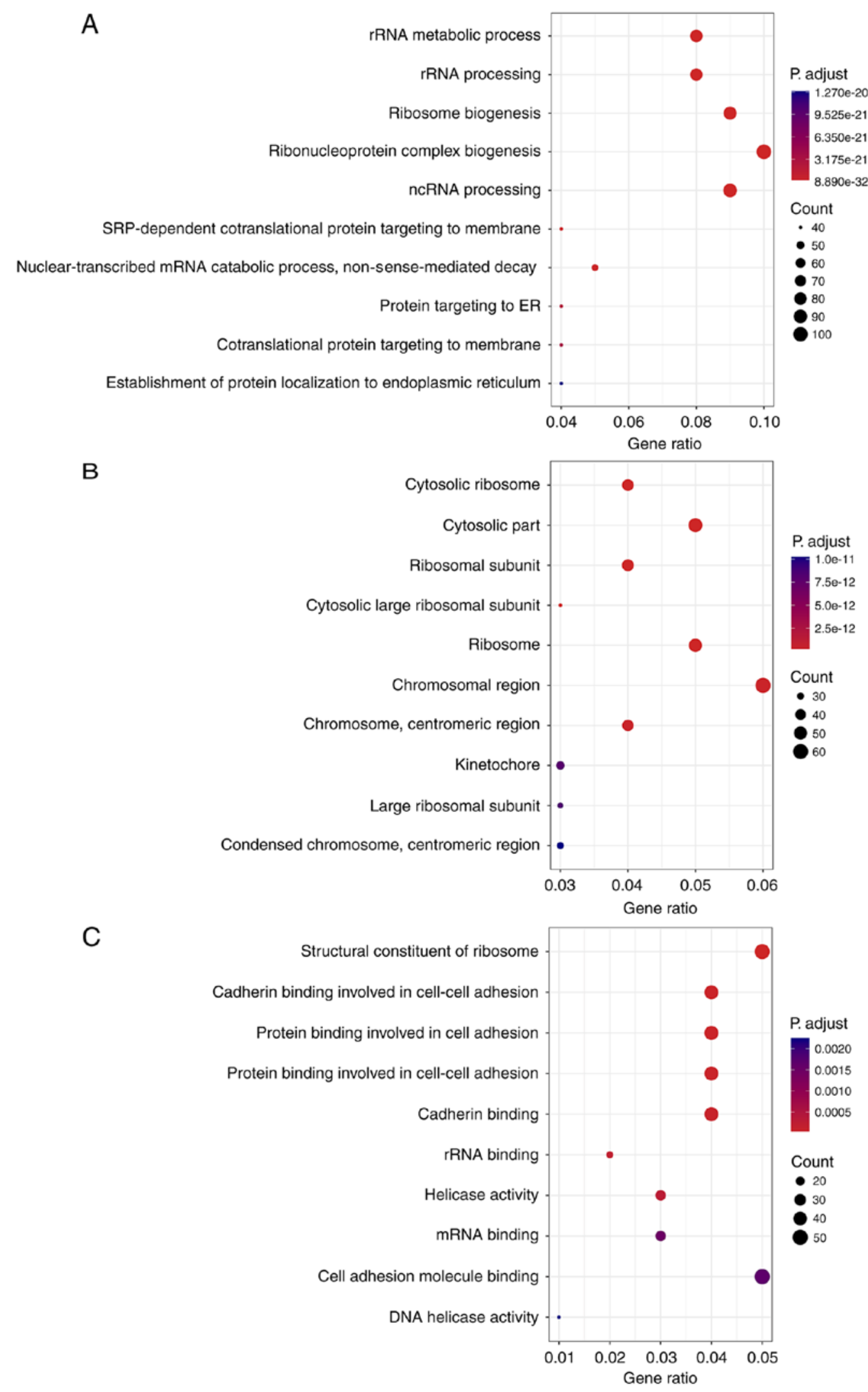

Figure 3. Gene ontology analysis of small nucleolar RNA-associated genes in the categories (A) biological process, (B) cellular component and (C) molecular function. rRNA, ribosomal RNA; ncRNA, non-coding RNA; ER, endoplasmic reticulum; SRP, signal recognition particle.

downregulated in HCC tissues and the remaining snoRNAs were significantly upregulated in HCC tissues (Fig. 5).

The HCC patients were divided into a high-risk group $(n=165)$ and a low-risk group $(n=165)$ according to the threshold of the median PI value (Fig. 6A). The patients were followed up for a duration of 91-3,675 days. The dependence of the overall survival status (dead or alive) on the snoRNA-based risk scores of the HCC patients was also plotted, displaying 
A

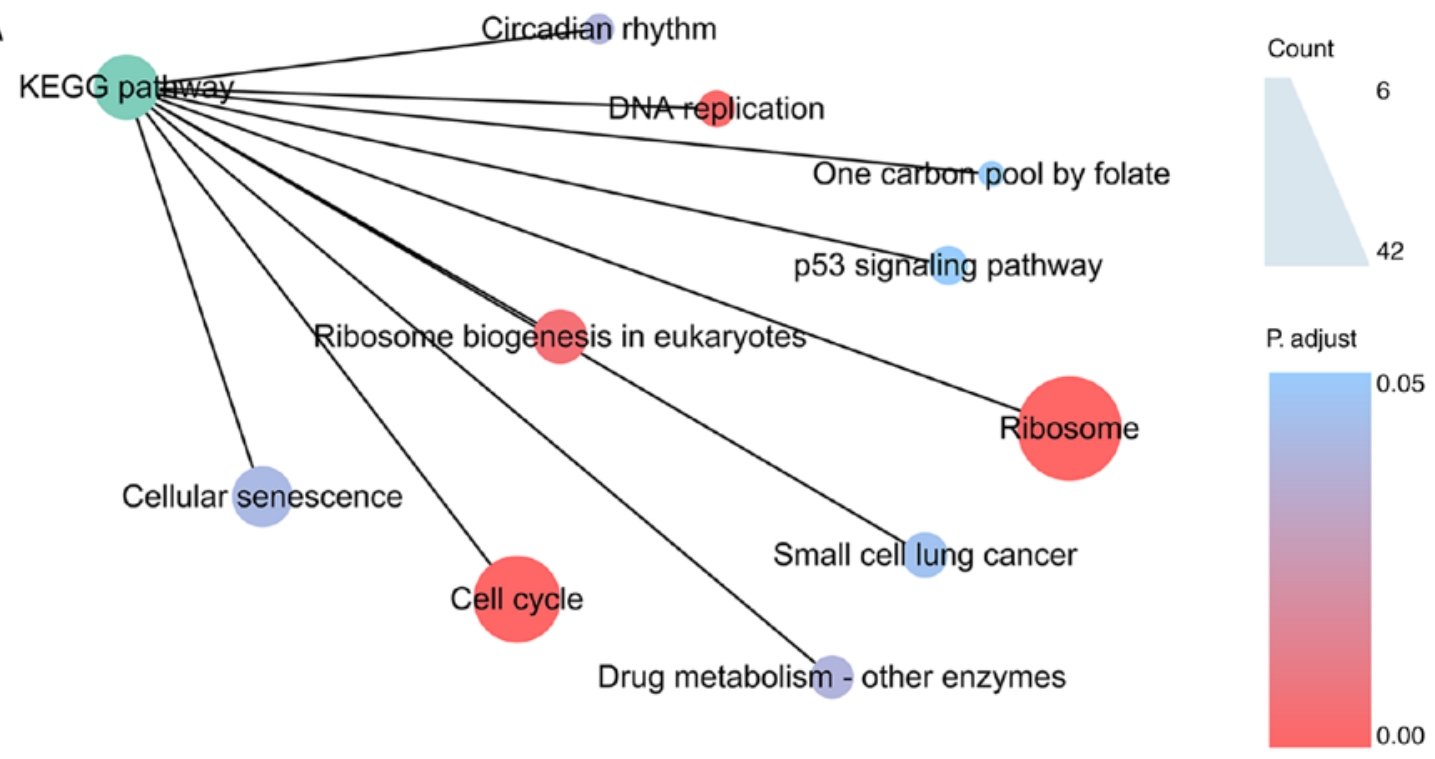

B Ribosome

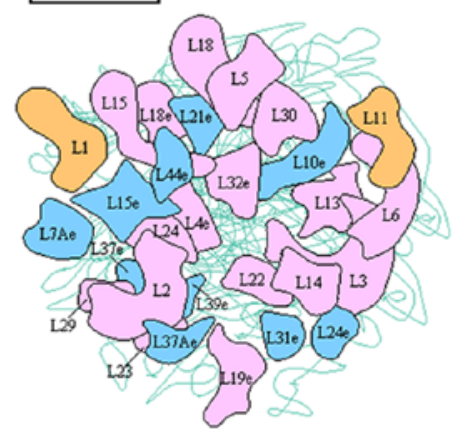

Large subunit (Haloarcula marismortui)

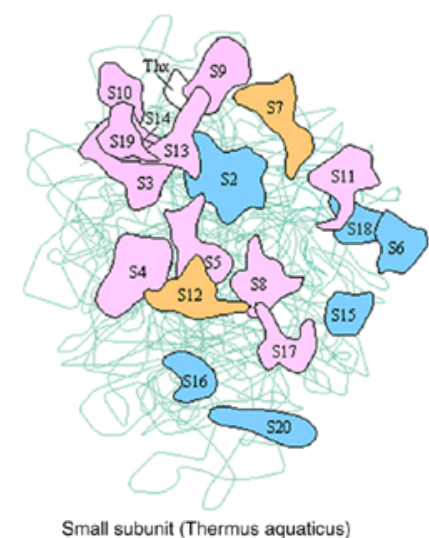

Ribosomal RNAs

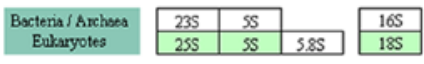

Ribosomal proteins
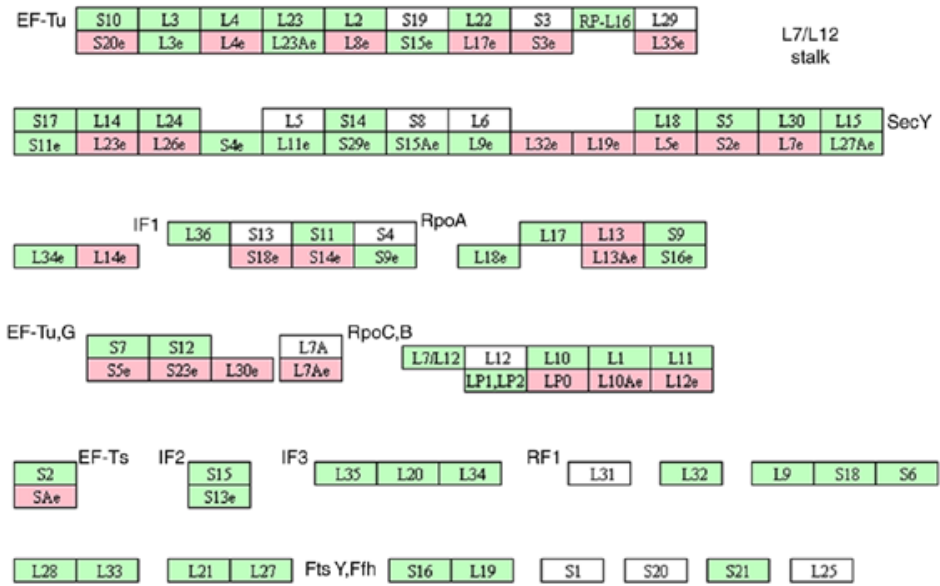

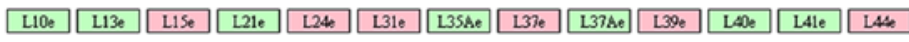

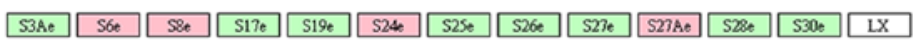

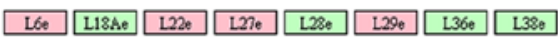

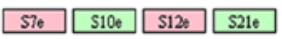

Figure 4. KEGG pathway analysis of small nucleolar RNA-associated genes. (A) Top 10 significantly enriched KEGG pathways. All pathways were determined based on the 'Homo sapiens' category of the KEGG database. (B) Ribosome was the most significant KEGG pathway (https://www.kegg.jp/dbget-bin/www_ bget?pathway+hsa03010). KEGG, Kyoto Encyclopedia of Genes and Genomes.

inferior survival for patients in the high-risk group (Fig. 6). Patients in the high-risk group had a significantly shorter median survival time than those in the low-risk group (hazard ratio=2.778, 95\% confidence interval: 1.904-4.051, $\mathrm{P}<0.001$; Fig. 7A). This result indicated the patients in the high-risk group have a 2.78-fold increased risk of death compared with those in the low-risk group. The area under the receiver operating characteristic curve was 0.731 , which indicated a moderate survival prediction ability of the PI (Fig. 7B). In the multivariate analysis (Table II), the risk model/PI that was proposed was demonstrated to be an independent prognostic factor, suggesting its independent prognostic value.

Deregulated pathways between high-and low-risk groups. To identify disturbed biological signaling pathways between the high- and low-risk groups, GSEA analysis was performed. Among all of the pre-defined KEGG pathway-associated gene sets, spliceosome, cell cycle and DNA replication signaling pathways were identified to be significantly linked with the survival risk estimated by the PI (Fig. 8), suggesting that 
A

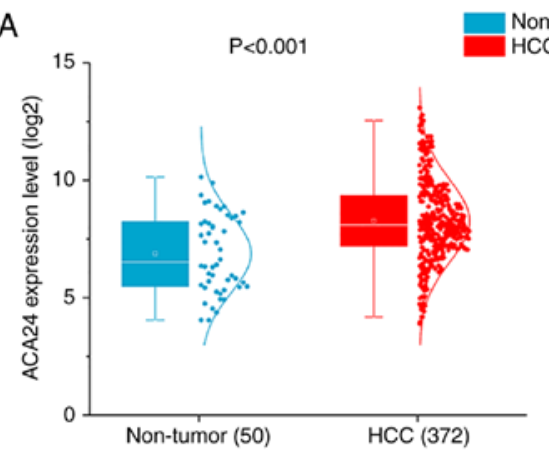

C

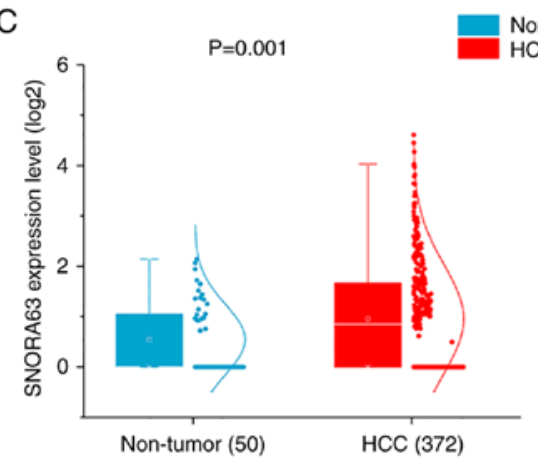

E

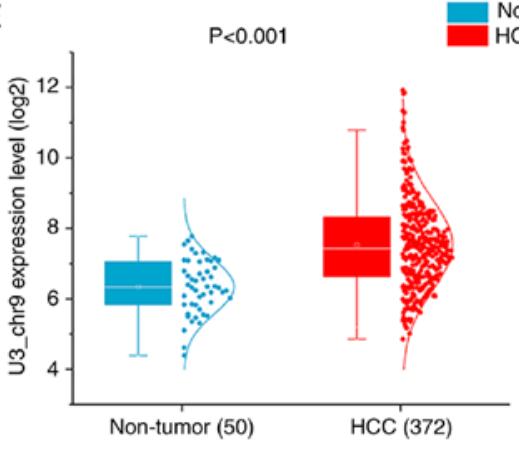

B

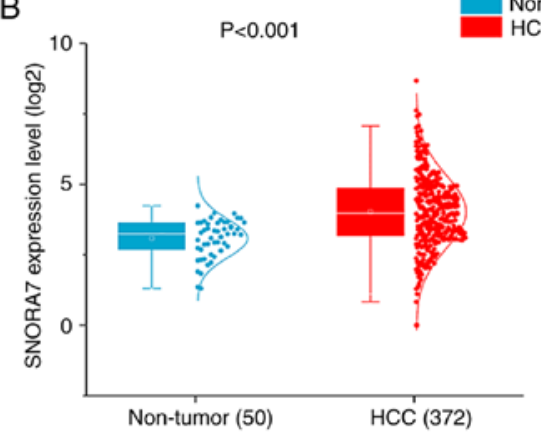
Non-tumor
HCC

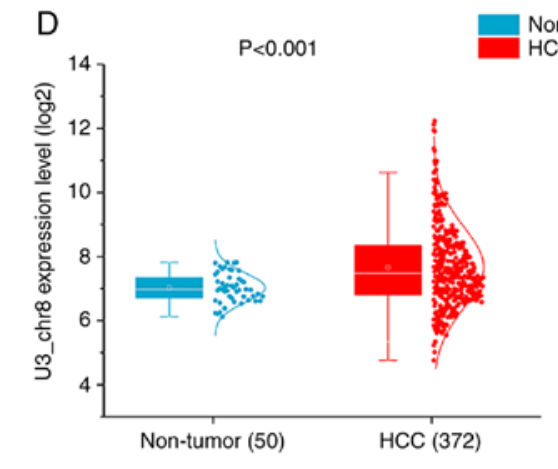

$\mathrm{F}$

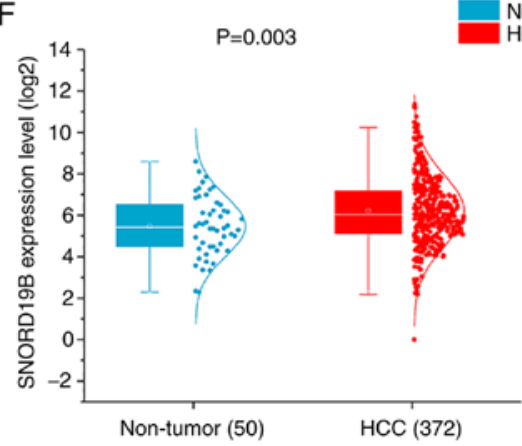

G

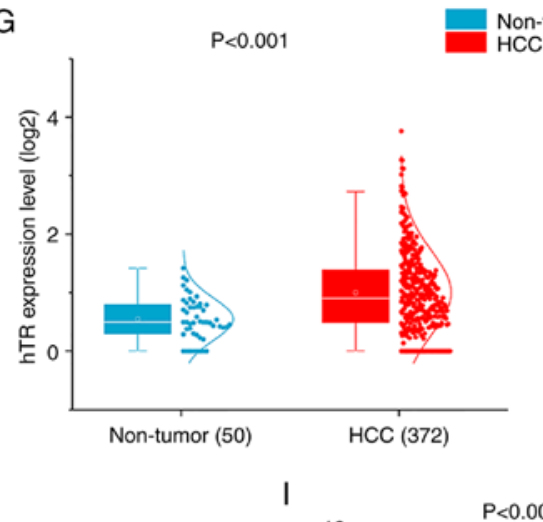

Non-tumor
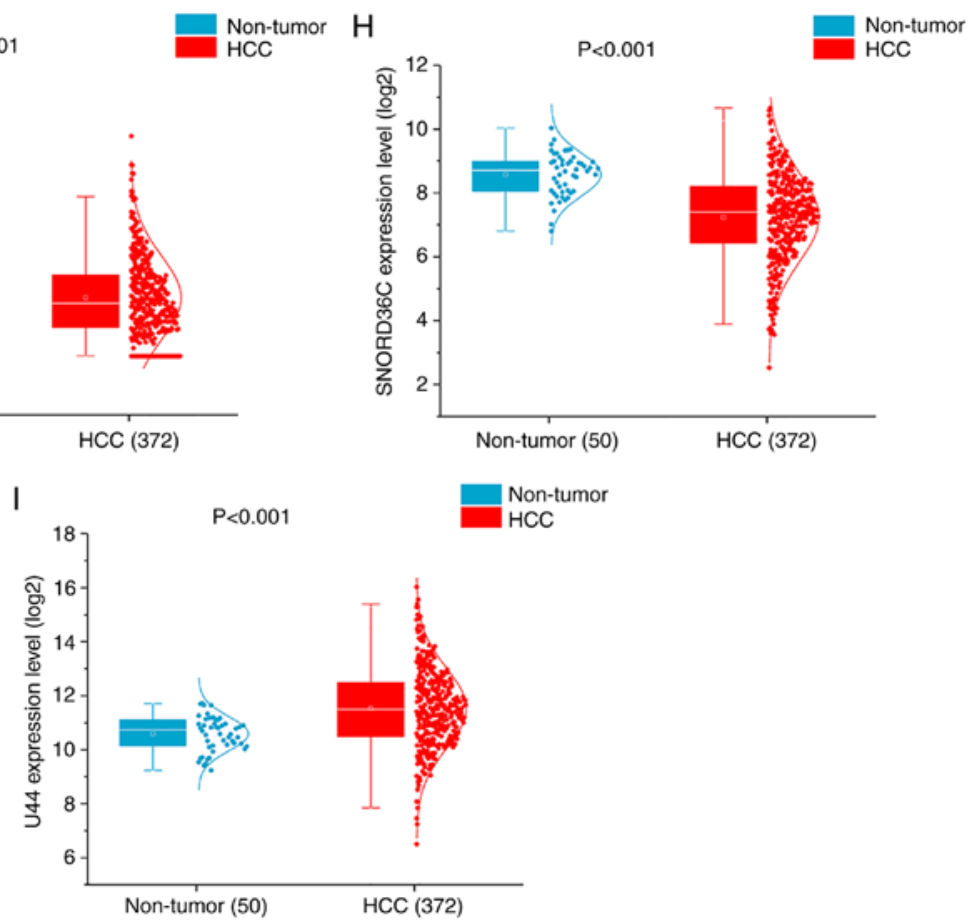

Figure 5. Differential expression of the 9 key snoRs between HCC and non-tumor tissues. White lines represent median value and white blocks represent the mean value. Boxes indicate the range of 25 to 75\%. (A) SNORA24; (B) SNORA7; (C) SNORA63; (D) U3_chr8-2; (E) U3_chr9; (F) SNORD19B; (G) hTR; (H) SNORD36C; (I) SNORD44. HCC, hepatocellular carcinoma; SNOR, small nucleolar RNA; chr, chromosome; SNORA, SNOR, H/ACA box; SNORD, SNOR, C/D box. 
Table II. Univariate and multivariate analyses of factors affecting the overall survival of hepatocellular carcinoma patients from The Cancer Genome Atlas by Cox regression analysis.

\begin{tabular}{llrllll}
\hline & \multicolumn{2}{c}{ Univariate analysis } & & \multicolumn{2}{c}{ Multivariate analysis } \\
\cline { 2 - 2 } Variables & Hazard ratio (95\%CI) & P-value & & Hazard ratio (95\%CI) & P-value \\
\hline Age, years & & & & & \\
$(\geq 60$ vs. $<60$ years) & $1.045(0.717-1.523)$ & 0.818 & & \\
Gender (male vs. female) & $0.875(0.593-1.292)$ & 0.503 & & \\
Pathological stage (I-II vs. III-IV) & $2.964(1.978-4.440)$ & $<0.001$ & & $1.392(0.188-10.297)$ & 0.746 \\
Tumor stage (T1-T2 vs. T3-T4) & $3.028(2.070-4.427)$ & $<0.001$ & & $1.714(0.231-12.705)$ & 0.598 \\
Lymph node metastasis (positive vs. negative) & $2.507(0.611-10.287)$ & 0.202 & & \\
Distant metastasis (positive vs. negative) & $4.911(1.536-15.701)$ & 0.007 & & $1.395(0.329-5.909)$ & 0.652 \\
Histological grade (G1-G2 vs. G3-G4) & $1.098(0.743-1.623)$ & 0.640 & & & \\
Tumor status (with tumor vs. tumor free) & $3.610(2.322-5.613)$ & $<0.001$ & & $3.313(1.940-5.659)$ & $<0.001$ \\
Vascular tumor cell type (micro+macro vs. none) & $1.352(0.852-2.146)$ & 0.200 & & & \\
Prognostic index (high vs. low risk) & $2.853(1.903-4.278)$ & $<0.001$ & & $3.023(1.785-5.119)$ & $<0.001$ \\
\hline
\end{tabular}

CI, confidence interval.
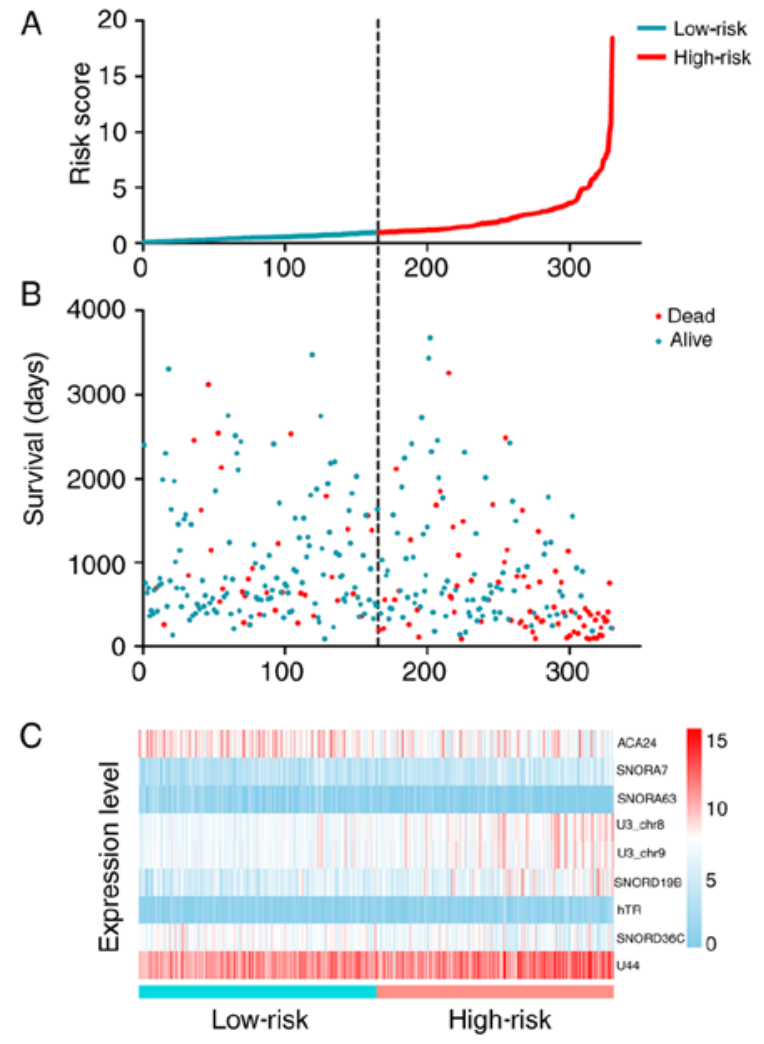

Figure 6. snoRNA risk score analysis for HCC patients. (A) Stratification of HCC patients in the risk score vs. snoRNA signature plot into low- and high-risk score groups. The $\mathrm{x}$-axis displays the order of patients based on risk score and the y-axis the snoRNA signature. (B) Survival status (dead or alive) and overall survival time of $\mathrm{HCC}$ cases with different expression of the signature snoRNAs. The patients were followed up for a duration of 91-3,675 days. The $\mathrm{x}$-axis displays the order of patients based on risk score and $\mathrm{y}$-axis the survival time of patients. (C) Heatmap of the included snoRNAs in the prognostic signature. snoRNA, small nucleolar RNA; HCC, hepatocellular carcinoma.

patients in the high-risk group may have inferior survival due to the above cancer-associated signaling pathways.

\section{Discussion}

Patients with HCC are at a substantial risk of metastasis, recurrence and death, although the treatment methods have markedly improved. A deeper understanding of the molecular mechanisms is required to develop appropriate treatment protocols and promote precision medicine. The present study comprehensively analyzed the expression profiles of snoRNAs in $\mathrm{HCC}$ and identified an overall elevation in the expression of certain snoRNAs. Furthermore, the potential functional terms and pathways of snoRNAs were determined, which mainly involved ribosome-associated processes and the cell cycle. Considering the indispensable function of certain snoRNAs in HCC, a prognostic method based on 9 snoRNAs was developed to stratify HCC patients into subgroups with different risks of mortality. Based on the GSEA analysis, disruption of the spliceosome may be the major contributor to the poor survival of patients in the high-risk group.

Hepatocarcinogenesis is considered a multi-step process, with various molecular factors, including snoRNAs, involved in its development and progression. To date, only few studies that have delineated the clinical significance and molecular mechanisms of snoRNAs in HCC. Hence, the present study comprehensively investigated the expression profiles of snoRNAs in HCC and observed an overall upregulation of snoRNAs in HCC tissues. Several HCC-associated oncogenic snoRNAs, which are upregulated in HCC, have been previously reported, whereas downregulated snoRNAs may act as tumor suppressors. Several of the dysregulated snoRNAs identified in the present study were also reported in previous studies; for instance, Fang et al (14) indicated that SNORD 126 (chr14_20794608_20794685) was highly expressed in HCC compared with non-tumorous samples. They also identified that upregulated SNORD126 was associated with a shorter survival rate of HCC patients. However, these results were the opposite of the present results, according to which SNORD126 was downregulated in HCC tissues. The limited 

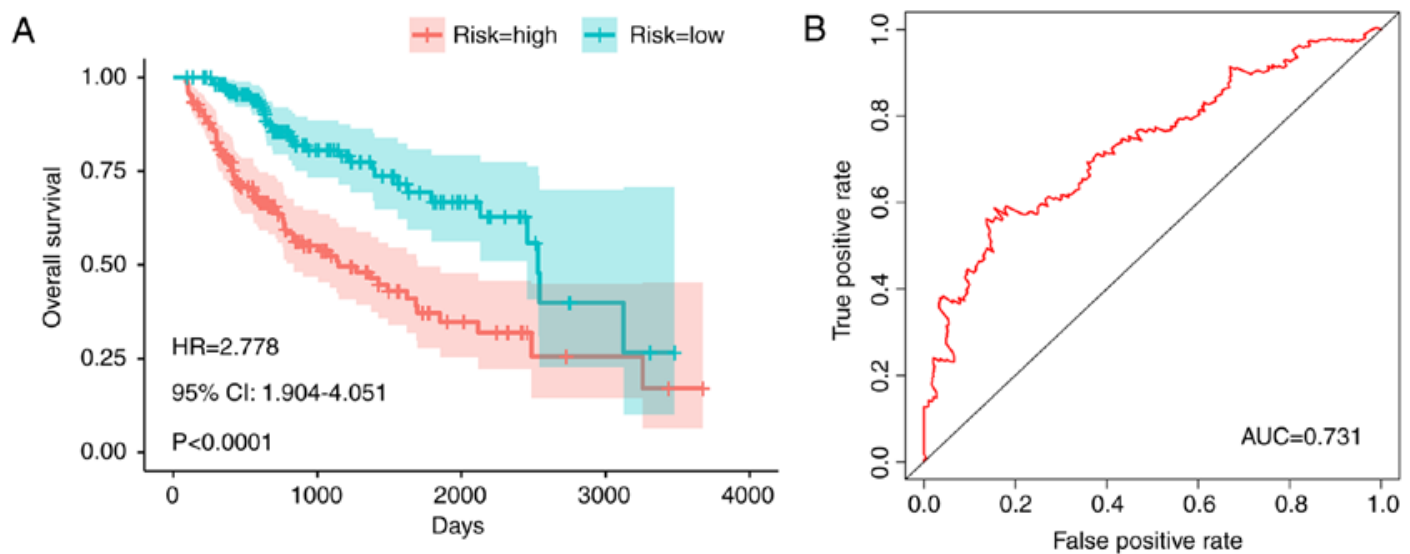

Figure 7. Kaplan-Meier and ROC curve analyses for the prognostic signature in HCC patients. (A) Kaplan-Meier survival curves displaying overall survival outcomes for patients rated as high- and low-risk according to the prognostic signature. Vertical lines indicate censored values. (B) Time-dependent ROC curve analysis for survival prediction based on the prognostic signature. ROC, receiver operating characteristic; HR, hazard ratio; AUC, area under curve; CI, confidence interval.

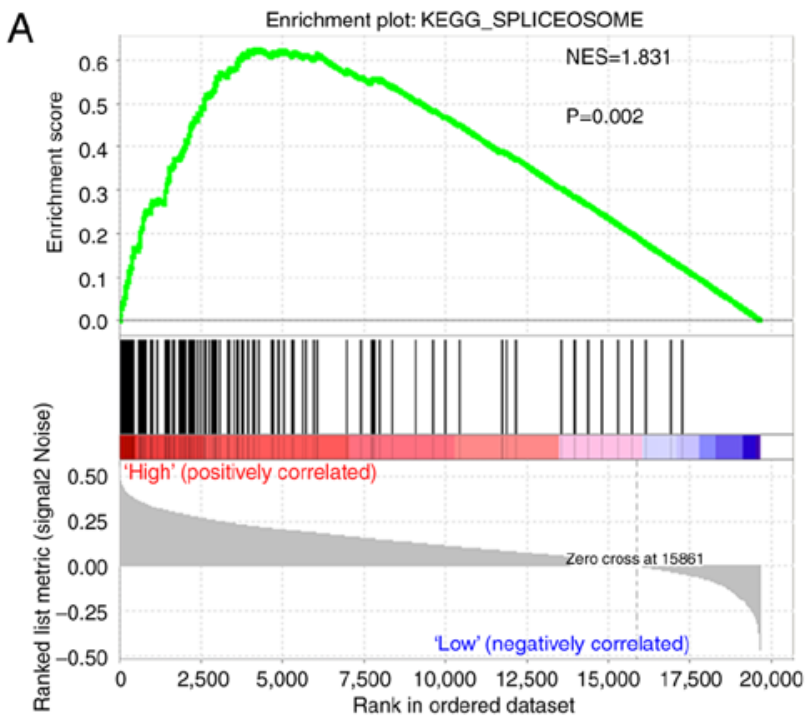

-Enrichment profile - Hits - - Ranking metric scores

B
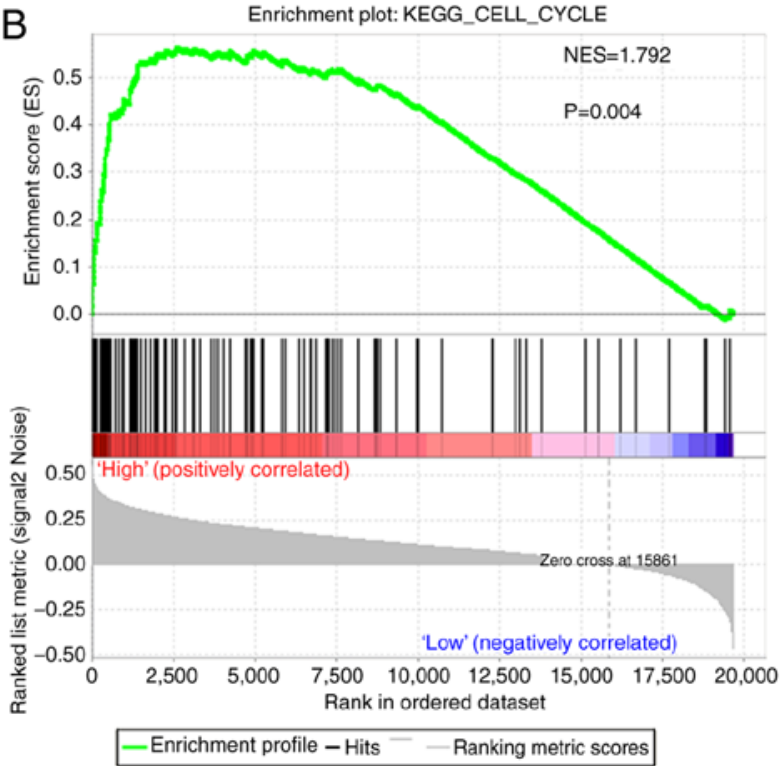

C Enrichment plot:KEGG_DNA_REPLICATION

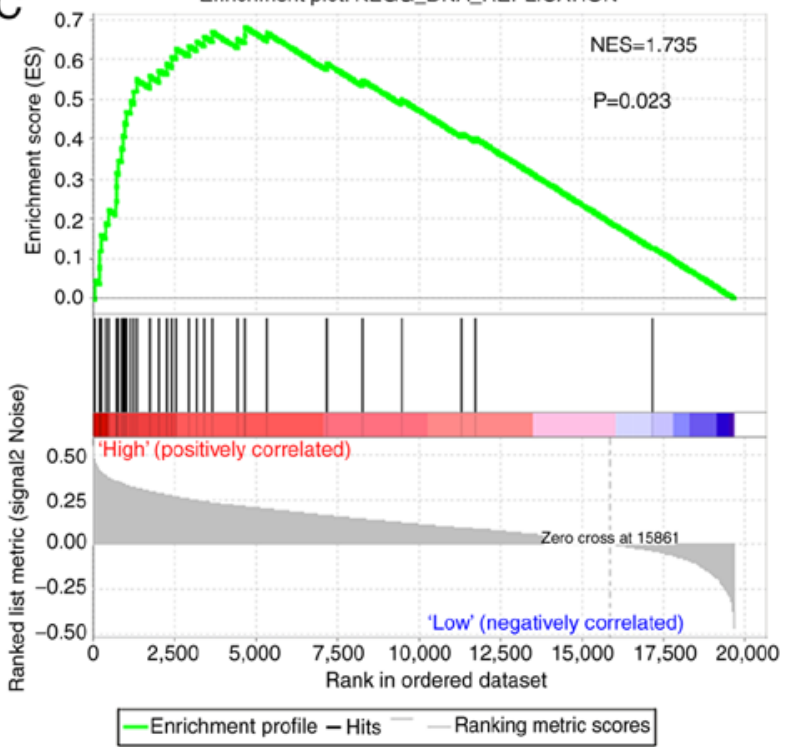

Figure 8. Gene set enrichment analysis of patients stratified into high- and low-risk group. (A) Spliceosome; (B) Cell cycle; (C) DNA replication. KEGG, Kyoto Encyclopedia of Genes and Genomes; NES, normalized enrichment score. 
number of cases in their study (only $30 \mathrm{HCC}$ tissues) may be the major reason for this difference. Wu et al (20) reported that the overexpression of SNORD76 is associated with decreased survival of HCC patients. In vitro and in vivo functional studies consistently indicated that SNORD76 promoted HCC cell growth and tumorigenicity. The high expression levels of another markedly upregulated snoRNA, SNORD78, has also been validated in HCC, and knockdown of SNORD78 significantly suppressed the proliferation, migration and invasion of liver tumor cells (21). These studies have facilitated a better understanding of the function of snoRNAs in HCC and provided novel ideas for early diagnosis and the development of precision medical treatments. The present analysis broadens the scope and promotes the search for novel snoRNAs as diagnostic and prognostic markers in HCC.

At present, the rudimentary understanding of the roles of snoRNAs in HCC limits their clinical application. Therefore, functional enrichment analysis was performed to determine the precise biological processes that were deregulated by the aberrant expression of snoRNAs in HCC. It was identified that snoRNAs may be involved in the pathways of ribosome structure and cell cycle, which indicated that snoRNAs significantly affect cell growth. Indeed, snoRNAs often combine with ribonucleoproteins (RNPs) to form stable and functional snoRNP particles, which is necessary for the effective and accurate formation of ribosomes (22). Ribosomes are considered to be the processing plants for protein synthesis in cells, but in tumor cells, this molecular machinery is misaligned and cellular metabolism is deregulated (23). Upregulated cell proliferation is usually accompanied by changes in the ribosome production rate. Perturbations of ribosome and ribosome-associated pathogenesis have been reported to be associated with multiple cancer types $(24,25)$. In HCC, several tumor suppressors and oncogenes have been identified to either affect the development of the mature ribosome or to regulate the activity of proteins (26). Therefore, dysregulated snoRNAs presumably exert an oncogenic or tumor suppressor function and may regulate the malignant phenotype by altering the ribosome synthesis machinery.

The highlight of the present study is that it was the first, to the best of our knowledge, to propose a snoRNA-based prognostic signature for HCC patients. For a decade, TCGA has collected large-scale molecular profiles and clinicopathologic annotation data, which has made it possible to identify key features that determine the clinical outcome of HCC patients (17). Identifying the distinct molecular features of each tumor patient makes it possible to lay a foundation for the development of personalized medicine (27). Several previous studies have proposed molecular prognostic signatures based on the expression levels of IncRNAs (28), miRNAs (29) and mRNAs (30). However, a snoRNA-based risk score has not been described to the best of our knowledge. snoRNAs are stable and measurable in peripheral plasma and serum, which gives snoRNAs a unique advantage as potential molecular biomarkers for the diagnosis and prognosis of tumor patients $(11,31)$.

To the best of our knowledge, the present study was the first to propose a prognostic signature based on snoRNAs, which had a satisfactory ability to predict survival. The present study also improved the current understanding of the molecular mechanisms of HCC. The prospective molecular mechanisms of the key deregulated snoRNAs were also assessed. Of note, abnormal alternative splicing events may be the cause for the adverse clinical outcomes for patients with a high prognostic index in the high-risk group. Of note, several snoRNAs have been reported to have a role in pre-mRNA splicing (32). However, the key snoRNAs identified in the present study have not been reported. Hence, the specific regulatory mechanisms of snoRNAs in splicing in HCC should be further explored in the future.

In summary, the present study was the first to propose a prognostic signature based on 9 snoRNAs in HCC, each of which is an independent risk factor. Numerous genes with statistically significant prognostic associations were identified for further study. These snoRNAs may be utilized as novel therapeutic targets or molecular markers for HCC with high clinical significance. The results of the present in silico analysis should be verified by in vivo and in vitro experiments in the future. The potential functional terms and molecular pathways of mRNAs associated with the snoRNAs were also assessed. The prognostic signature established in the present study may be a clinically useful tool that is easily incorporated into a clinical RNA-sequencing program to individualize HCC therapy.

\section{Acknowledgements}

Not applicable.

\section{Funding}

The present study was supported in part by the Fund of the National Natural Science Foundation of China (grant nos. NSFC81060202, NSFC81860319 and NSFC81260222), the Guangxi Science Technology Program (grant no. GuikeAB17195020), the Innovation Project of Guangxi Graduate Education (grant no. YCSW2018104), the Guangxi Medical University Training Program for Distinguished Young Scholars and the Medical Excellence Award Funded by the Creative Research Development Grant of the First Affiliated Hospital of Guangxi Medical University.

\section{Availability of data and materials}

The datasets used and/or analyzed during this study are available from the corresponding author on reasonable request.

\section{Authors' contributions}

The study was designed by GC, YWD, YH and HY. PL, HY, HYW, HYL and YWD performed the statistical analysis. PL and HY wrote the draft and GC, HY, YH and YWD corrected the manuscript. All authors read and approved the final manuscript.

\section{Ethics approval and consent to participate}

Not applicable.

\section{Patient consent for publication}

Not applicable. 


\section{Competing interests}

The authors declare that they have no competing interests.

\section{References}

1. Siegel RL, Miller KD and Jemal A: Cancer statistics, 2018. CA Cancer J Clin 68: 7-30, 2018.

2. Forner A, Reig M and Bruix J: Hepatocellular carcinoma. Lancet 391: 1301-1314, 2018.

3. Fu $\mathrm{J}$ and Wang $\mathrm{H}$ : Precision diagnosis and treatment of liver cancer in China. Cancer Lett 412: 283-288, 2018.

4. Bosetti C, Turati F and La Vecchia C: Hepatocellular carcinoma epidemiology. Best Pract Res Clin Gastroenterol 28: 753-770, 2014.

5. Sherman M: Hepatocellular carcinoma: Epidemiology, surveillance, and diagnosis. Semin Liver Dis 30: 3-16, 2010.

6. Kudo M: Systemic therapy for hepatocellular carcinoma: 2017 update. Oncology 93 (Suppl 1): S135-S146, 2017.

7. Costentin CE, Ferrone CR, Arellano RS, Ganguli S, Hong TS and Zhu AX: Hepatocellular carcinoma with macrovascular invasion: Defining the optimal treatment strategy. Liver Cancer 6 : 360-374, 2017

8. Kissel M, Berndt S, Fiebig L, Kling S, Ji Q, Gu Q, Lang T, Hafner FT, Teufel M and Zopf D: Antitumor effects of regorafenib and sorafenib in preclinical models of hepatocellular carcinoma. Oncotarget 8: 107096-107108, 2017.

9. Sherman M: Regorafenib for treatment of hepatocellular carcinoma. Hepatology 67: 1162-1165, 2018.

10. Bruix J, Qin S, Merle P, Granito A, Huang YH, Bodoky G, Pracht M, Yokosuka O, Rosmorduc O, Breder V, et al: Regorafenib for patients with hepatocellular carcinoma who progressed on sorafenib treatment (RESORCE): A randomised, double-blind, placebo-controlled, phase 3 trial. Lancet 389: 56-66, 2017.

11. Romano G, Veneziano D, Acunzo M and Croce CM: Small non-coding RNA and cancer. Carcinogenesis 38: 485-491, 2017.

12. Wang J, Samuels DC, Zhao S, Xiang Y, Zhao YY and Guo Y: Current research on non-coding ribonucleic acid (RNA). Genes 8: pii: E366, 2017.

13. Xu G, Yang F, Ding CL, Zhao LJ, Ren H, Zhao P, Wang W and Qi ZT: Small nucleolar RNA 113-1 suppresses tumorigenesis in hepatocellular carcinoma. Mol Cancer 13: 216, 2014.

14. Fang X, Yang D, Luo H, Wu S, Dong W, Xiao J, Yuan S, Ni A, Zhang KJ, Liu XY, et al: SNORD126 promotes HCC and CRC cell growth by activating the PI3K-AKT pathway through FGFR2. J Mol Cell Biol 9: 243-255, 2017.

15. Gong J, Li Y, Liu CJ, Xiang Y, Li C, Ye Y, Zhang Z, Hawke DH, Park PK, Diao L, et al: A Pan-cancer analysis of the expression and clinical relevance of small nucleolar RNAs in human cancer. Cell Rep 21: 1968-1981, 2017.

16. Yu G, Wang LG, Han Y and He QY: ClusterProfiler: An $\mathrm{R}$ package for comparing biological themes among gene clusters. OMICS 16: 284-287, 2012.

17. Liu J, Lichtenberg T, Hoadley KA, Poisson LM, Lazar AJ, Cherniack AD, Kovatich AJ, Benz CC, Levine DA, Lee AV, et al An integrated TCGA Pan-Cancer clinical data resource to drive High-quality survival outcome analytics. Cell 173: 400-416.e11, 2018.
18. Jacob H, Stanisavljevic L, Storli KE, Hestetun KE, Dahl O and Myklebust MP: A four-microRNA classifier as a novel prognostic marker for tumor recurrence in stage II colon cancer. Sci Rep 8: 6157,2018

19. Subramanian A, Tamayo P, Mootha VK, Mukherjee S, Ebert BL, Gillette MA, Paulovich A, Pomeroy SL, Golub TR, Lander ES, et al: Gene set enrichment analysis: A knowledge-based approach for interpreting genome-wide expression profiles. Proc Natl Acad Sci USA 102: 15545-15550, 2005.

20. Wu L, Chang L, Wang H, Ma W, Peng Q and Yuan Y: Clinical significance of C/D box small nucleolar RNA U76 as an oncogene and a prognostic biomarker in hepatocellular carcinoma. Clin Res Hepatol Gastroenterol 42: 82-91, 2018.

21. Ma P, Wang H, Han L, Jing W, Zhou X and Liu Z: Up-regulation of small nucleolar RNA 78 is correlated with aggressive phenotype and poor prognosis of hepatocellular carcinoma. Tumour Biol: Oct 21, 2016 (Epub ahead of print)

22. Reichow SL, Hamma T, Ferré-D'Amaré AR and Varani G: The structure and function of small nucleolar ribonucleoproteins. Nucleic Acids Res 35: 1452-1464, 2007.

23. Dong Z, Zhu C, Zhan Q and Jiang W: The roles of RRP15 in nucleolar formation, ribosome biogenesis and checkpoint control in human cells. Oncotarget 8: 13240-13252, 2017.

24. Su H, Xu T, Ganapathy S, Shadfan M, Long M, Huang TH, Thompson I and Yuan ZM: Elevated snoRNA biogenesis is essential in breast cancer. Oncogene 33: 1348-1358, 2014.

25. Russo A, Saide A, Smaldone S, Faraonio R and Russo G: Role of uL3 in multidrug resistance in p53-mutated lung cancer cells. Int J Mol Sci 18: pii: E547, 2017.

26. Yuan F, Zhang Y, Ma L, Cheng Q, Li G and Tong T: Enhanced NOLC1 promotes cell senescence and represses hepatocellular carcinoma cell proliferation by disturbing the organization of nucleolus. Aging Cell 16: 726-737, 2017.

27. Cristescu R, Lee J, Nebozhyn M, Kim KM, Ting JC, Wong SS Liu J, Yue YG, Wang J, Yu K, et al: Molecular analysis of gastric cancer identifies subtypes associated with distinct clinical outcomes. Nat Med 21: 449-456, 2015.

28. Wang Z, Wu Q, Feng S, Zhao Y and Tao C: Identification of four prognostic LncRNAs for survival prediction of patients with hepatocellular carcinoma. PeerJ 5: e3575, 2017.

29. Liu G, Wang H, Fu JD, Liu JY, Yan AG and Guan YY: A five-miRNA expression signature predicts survival in hepatocellular carcinoma. APMIS 125: 614-622, 2017.

30. Li B, Feng W, Luo O, Xu T, Cao Y, Wu H, Yu D and Ding Y: Development and validation of a three-gene prognostic signature for patients with hepatocellular carcinoma. Sci Rep 7: 5517, 2017.

31. Mannoor K, Liao J and Jiang F: Small nucleolar RNAs in cancer. Biochim Biophys Acta 1826: 121-128, 2012.

32. Warner WA, Spencer DH, Trissal M, White BS, Helton N, Ley TJ and Link DC: Expression profiling of snoRNAs in normal hematopoiesis and AML. Blood Adv 2: 151-163, 2018.

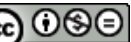

This work is licensed under a Creative Commons Attribution-NonCommercial-NoDerivatives 4.0 International (CC BY-NC-ND 4.0) License. 